\title{
Urinary microRNA-based signature improves accuracy of detection of clinically relevant prostate cancer within the prostate-specific antigen grey zone
}

\author{
ALBERTO IVAN SALIDO-GUADARRAMA ${ }^{1}$, JORGE GUSTAVO MORALES-MONTOR ${ }^{2}$, \\ CLAUDIA RANGEL-ESCAREÑO ${ }^{3}$, ELIZABETH LANGLEY ${ }^{4}$, OSCAR PERALTA-ZARAGOZA ${ }^{5}$, \\ JOSE LUIS CRUZ COLIN ${ }^{1}$ and MAURICIO RODRIGUEZ-DORANTES ${ }^{1}$ \\ ${ }^{1}$ Oncogenomics Laboratory, The National Institute of Genomic Medicine, Mexico City 14610; \\ ${ }^{2}$ Urology Department, Hospital General Dr. Manuel Gea Gonzalez, Mexico City $14080 ;{ }^{3}$ Computational Genomics,
The National Institute of Genomic Medicine, Mexico City 14610; ${ }^{4}$ Department of Basic Research,
National Institute of Cancerology, Mexico City $14080 ;{ }^{5}$ Division of Chronic Infections and Cancer,
Research Center in Infection Diseases, National Institute of Public Health, Cuernavaca 62100, Mexico
}

Received August 31, 2015; Accepted February 15, 2016

DOI: $10.3892 / \mathrm{mmr} .2016 .5095$

\begin{abstract}
At present, prostate-specific antigen (PSA) is used as a clinical biomarker for prostate cancer $(\mathrm{PCa})$ diagnosis; however, a large number of patients with benign prostate hyperplasia (BPH) with PSA levels in the 'gray area' $(4-10 \mathrm{ng} / \mathrm{ml})$ are currently subjected to unnecessary biopsy due to overdiagnosis. Certain microRNAs (miRs) have been proven to be useful biomarkers, several of which are detectable in bodily fluids. The present study identified and validated a urinary miR-based signature to enhance the specificity of PCa diagnosis and to reduce the number of patients with benign conditions undergoing biopsy. Seventy-three urine samples from Mexican patients with diagnosis of PCa with a Gleason score $\geq 7$ and 70 patients diagnosed with BPH were collected after digital rectal examination (DRE) of the prostate. miR expression profiles were determined using TaqMan Low Density Array experiments, and normalized Ct values for the miRs were compared between PCa and BPH groups. Receiver operating characteristic (ROC) curve analysis was performed to evaluate whether miR detection in urine is suitable for distinguishing patients with PCa from those with BPH. The identified miR-100/200b signature was significantly correlated with $\mathrm{PCa}$. Using a multivariable logistic regression approach, a base model including the clinical variables age, prostate-specific antigen (PSA), the percentage of free PSA
\end{abstract}

Correspondence to: Dr Mauricio Rodriguez-Dorantes, Oncogenomics Laboratory, The National Institute of Genomic Medicine, 4809 Periférico Sur, Arenal Tepepan, Tlalpan, Mexico City 14610, Mexico

E-mail: mrodriguez@inmegen.gob.mx

Key words: microRNA, prostate cancer, prostate-specific antigen grey zone, urine and DRE was generated, and a second base model additionally contained the miR-100/200b signature. ROC analysis demonstrated that the combined model significantly outperformed the capacity of PSA $(\mathrm{P}<0.001)$ and the base model $(\mathrm{P}=0.01)$ to discriminate between $\mathrm{PCa}$ and $\mathrm{BPH}$ patients. In terms of evaluation of the sub-group of patients in the gray zone of PSA levels, the performance of the combined model for predicting $\mathrm{PCa}$ cases was significantly superior to PSA level determination $(\mathrm{P}<0.001)$ and the base model $(\mathrm{P}=0.009)$. In addition, decision curve analysis demonstrated that the use of the combined model increased the clinical benefit for patients and produced a substantial reduction in unnecessary biopsies across a range of reasonable threshold probabilities $(10-50 \%)$. Detection of the urinary miR signature identified in the present study as part of clinical diagnostic procedures will enhance the accuracy of PCa diagnosis and provide a clinical benefit for patients with BPH by sparing them from undergoing invasive biopsy. To the best of our knowledge, the present study was the first to describe the profiling of urinary miR100 and miR-200b levels for the clinical diagnosis of PCa.

\section{Introduction}

Prostate cancer (PCa) is the second most frequently diagnosed malignancy and the sixth leading cause of cancer-associated mortality in men worldwide (1). It develops mainly in the peripheral zone and involves multiple genetic and molecular alterations (2). Prostate-specific antigen (PSA) remains the only diagnostic biomarker and indicator for the management of PCa. Inclusion of PSA evaluation into clinical assessments has achieved a significant reduction in $\mathrm{PCa}$-associated mortality (3). Although highly tissue-specific, PSA is not disease-specific (4). As a consequence, PSA routine screening has led to overdiagnosis and overtreatment, and controversies have risen regarding the increasing number of negative, potentially unnecessary prostate biopsies performed in order to identify a patient with a lethal prostate tumor (5). 
Considerable research efforts have been made with the aim of discovering novel molecular markers for improving $\mathrm{PCa}$ detection and monitoring (6). MicroRNAs (miRNAs) have emerged as promising diagnostic biomarkers and as therapeutic targets for a diversity of tumor types. Specific miRNAs have been found to have key regulatory roles in a variety of oncogenic processes, including angiogenesis (7), response to hypoxia (8), epithelial-mesenchymal transition (9) and metastasis $(10,11)$. Of note, a variety of studies have described the deregulation of several miRNAs during the genesis and progression of PCa (12). For example, miR-15a, miR-16-1, miR-101, the miR-200 family, miR-205, and the miR-99 family haven been shown to have tumor suppressor roles, while miR-21, miR-221/222, miR-106b-25-93 and miR-125b have been validated to have oncogenic functions (12). In addition, profiling studies of tissue samples have identified PCa-specific miRNA signatures, which permit the differentiation of malignant and benign conditions $(13,14)$.

It is of great interest to develop diagnostic strategies that are less invasive while being affordable. In this context, it is desirable to detect biomarkers in bodily fluids. miRNAs are relatively stable molecules and can be secreted into the extracellular space (15), which renders them useful biomarkers, readily detectable in bodily fluids. It has been shown that blood (16,17), saliva (18) and urine (19-22) contain miRNAs that can be quantified using methods such as reverse-transcription quantitative polymerase chain reaction (RT-qPCR). The most successful example is the case of the urine-based method for detecting and measuring levels of the non-coding transcript PCA3 for the diagnosis of PCa (23). In the present study, a high-throughput analysis of miRNAs in urine samples from men with PCa and benign prostate hyperplasia (BPH) was performed. A miRNA signature characteristic for $\mathrm{PCa}$ was identified and its clinical applicability for predicting the biopsy outcome of patients with suspected PCa was assessed.

\section{Materials and methods}

Patient cohort. A total of 143 male Mexican patients, who visited the Hospital General Dr. Manuel Gea Gonzalez (Mexico City, Mexico) between July 2012 and February 2013, were enrolled in the present study. Written informed consent was obtained from all patients. The protocol of the present study was approved by the ethics committee of Hospital General Dr. Manuel Gea Gonzalez. All patients presented with palpable nodules or induration of the prostate [suspicious digital rectal examination (DRE)] and blood levels of PSA $>2.5 \mathrm{ng} / \mathrm{ml}$. Following diagnosis, patients where scheduled for a 12-core transrectal ultrasound-guided prostate biopsy. Subsequently, an experienced pathologist assessed the cores to determine whether the biopsy specimens were malignant. For those samples pathologically diagnosed as PCa, Gleason scoring was performed by careful examination of the microscopic pattern of the cancer foci. The PCa group included 73 patients with significant PCa diagnosis (Gleason score $\geq 7$ ). The control group comprised 70 patients with $\mathrm{BPH}$, whose biopsy specimens contained benign glands and normal prostate cells only. Patients with previous diagnosis of PCa or a family history record of $\mathrm{PCa}$ were excluded. Patients with symptoms of urinary tract infection, immunodeficiency disorders, harboring high-grade prostatic intraepithelial neoplasia or atypic cellular proliferation, or who were under medical treatment for prostate hyperplasia, were also excluded. The clinical and pathological data of the patients were collected and are listed in Table I.

Urine sample processing and RNA extraction. First-catch urine (30 $\mathrm{ml})$ was collected following a prostate massage to stimulate the release of prostate fluid into the urine, as described in a previous study (24). The collected urine and prostate fluid mixture was mixed with $5 \mathrm{ml}$ RNAlater buffer (Qiagen, Hilden, Germany) as an RNA stabilizing agent. All samples were stored at $-80^{\circ} \mathrm{C}$ for one week. For further processing, samples were defrosted on ice and then centrifuged at 5,000 x g. Once the supernatant was discarded, the urine sediment was washed twice with phosphate-buffered saline at $\mathrm{pH} 7.2$ and RNA extraction was performed using the RNeasy Mini kit (Qiagen, Inc.) according to the manufacturer's guidelines. RNA was re-suspended in $15 \mu \mathrm{l}$ RNAase-free water and quantified spectrophotometrically using a NanoDrop ND-1000 system (Thermo Fisher Scientific, Inc., Waltham, MA, USA).

cDNA preparation, pre-amplification and RT-qPCR analysis. For the screening study, RT was performed for each sample using the TaqMan ${ }^{\circledR}$ MicroRNA reverse transcription kit and the Megaplex ${ }^{\mathrm{TM}}$ RT primers, human pool A v2.0 (Thermo Fisher Scientific, Inc.). Subsequently, the newly synthesized complementary DNA was subjected to a pre-amplification step using the 2X Preamp Master Mix and the Megaplex 10X preamp primer mix (Thermo Fisher Scientific, Inc.). The product was then loaded onto a Human MicroRNA TaqMan Low Density Array (TLDA) set card A v.2.1 (Thermo Fisher Scientific, Inc.) and qPCR was performed using a 7900HT Fast Real-Time PCR system (Thermo Fisher Scientific, Inc.). RT, pre-amplification and $\mathrm{qPCR}$ reactions were performed according to manufacturer's instructions.

For the validation study and time course analysis, RT, pre-amplification and qPCR reactions were performed. The expression of let-7c, miR-193b, miR-100, miR-200b, miR-200c, miR-20a, miR-204, miR-195, miR-150, miR-16, miR-21 and let-7b was measured using TaqMan ${ }^{\circledR}$ MicroRNA Assays (cat. no. 4427975; Thermo Fisher Scientific, Inc.). For measurement of GAPDH and KLK3 mRNA expression levels, Single Tube TaqMan ${ }^{\circledR}$ Gene Expression Assays (cat. no. 4331182; Thermo Fisher Scientific, Inc.) were used.

Time-course analysis. After thawing, six samples of urine, including three $\mathrm{PCa}$ and three $\mathrm{BPH}$, were randomly selected from the entire cohort, and $10 \mathrm{ml}$ from each sample were pooled. The pool was maintained at $25^{\circ} \mathrm{C}$ and aliquots were removed in triplicate at $0,1,2,4,6,12$ and $24 \mathrm{~h}$ and placed at $4^{\circ} \mathrm{C}$. Once this time-series had been completed, RNA extraction, RT and pre-amplification reactions were performed for all aliquots. Quantification of miR-15a, miR-21, KLK3 and GAPDH was performed by qPCR, as described above. Comparisons between raw $\mathrm{Ct}$ distributions were assessed performed using the Friedman test. $\mathrm{P}<0.05$ was considered statistically significant.

Evaluation of human miRNA TLDA and RT-qPCR data. In order to avoid group bias, the cycle threshold $(\mathrm{Ct})$ distributions for all PCa and BPH samples were determined and a filtering 
procedure was applied to classify the miRNAs according to their level of detectability. A Ct value of 35 was set arbitrarily; miRNAs with $\mathrm{Ct}<35$ were considered as detected and those with $\mathrm{Ct}>35$ were considered undetected. miRNAs that were undetected in $>2$ samples for each group were removed from the analysis. Filtered raw $\mathrm{Ct}$ data were normalized using a rank-invariant set normalization procedure. Briefly, this normalization computes all rank-invariant sets of features between pair-wise comparisons of each samples against a pseudo-mean. The rank-invariant features were used as a reference for generating a smoothing curve, which was then applied to the entire sample. Differential expression of miRNAs was assessed using a Mann-Whitney U test with consideration of two different distributions for $\mathrm{BPH}$ and $\mathrm{CaP}$ groups and a Benjamini Hochberg test was applied to control the rate of false-positive results. miRNAs with a fold change $>2$ and a false discovery rate (FDR) adjusted to $\mathrm{P}<0.05$ were considered to be statistically significant. For individual assays, the comparative $\mathrm{Ct}$ method was used to determine the differential expression of miRNAs (25). $\mathrm{P}<0.05$ as assessed by a Mann-Whitney U test was considered to indicate a statistically significant difference. For miR-100 and miR-200b scores, the geometric mean of raw $\mathrm{Ct}$ values were calculated and the geometric mean of MammU6 and RNU48 was subtracted.

Statistical analysis. Univariable logistic regression was performed to assess the contribution of age, PSA, \% of free PSA, DRE and miR-100/200b score as individual predictors of PCa on biopsy results. Odds ratios (ORs) and corresponding 95\% confidence intervals $(95 \% \mathrm{CI})$ were computed for each predictor. Multivariable logistic regression was used to build a base model including the variables age, PSA, \% of free PSA and DRE, as well as a second combined model composed of the variables age, PSA, \% of free PSA, DRE and miR-100/200b score. ORs and $95 \%$ CI were also computed for each model. For evaluating the diagnostic performance of the individual variables, and the base and combined models, receiver-operating characteristic (ROC) curve analysis was carried out, and the areas under the curve (AUC) and corresponding 95\% CI were computed using the DeLong method. A $\chi^{2}$ test was applied to assess significant differences in the predictive value between ROC curves. To assess the clinical benefit of the models tested, a decision curve analysis (DCA) was performed, which illustrates the net benefit of a clinical intervention at various threshold probabilities, the minimum expected probability of a PCa diagnosis at which the physician in consultation with the patient opts for a biopsy. In brief, DCA assigns weight to the relative value of the benefits (true positives) versus the false positives. Data processing and statistical analyses were performed using utility packages from Bioconductor in $\mathrm{R}$ and GraphPad Prism V 5.0 (GraphPad, Inc., La Jolla, CA, USA).

\section{Results}

miRNAs are stable molecules which can be robustly measured in urine samples. As a proof of principle for the robustness of the approach of evaluating miRNAs as biomarkers in urine, the degradation pattern of selected miRNAs was determined. A time course RT-qPCR analysis of miRNAs in urine was performed at $37^{\circ} \mathrm{C}$. The levels of miR-16 and miR-21, two readily detectable miRNAs, were assessed in a variety of urine samples over $24 \mathrm{~h}$. Simultaneously, the levels of two mRNAs, GAPDH and KLK3, were assessed, which showed a rapid decrease over $24 \mathrm{~h}$. By contrast, the levels of the two miRNAs remained relatively constant (Fig. 1A). To test the reproducibility of the method for measuring miRNA levels, aliquots were taken in duplicate from the urine samples of two PCa patients and one BPH patient and the levels of miRNA expression were assessed by RT-qPCR in triplicate. The $\mathrm{Ct}$ values obtained showed no significant differences among replicates from the same sample donor and between samples from different donors (Fig. 1B). These results indicated that miRNAs are stable molecules in urine samples, which can be detected and robustly measured.

miRNA profile is significantly altered in PCa. A PCR array was performed to determine the levels of 364 miRNAs in 8 urine samples from patients with $\mathrm{PCa}$ and 8 urine samples from patients with BPH. Notably, miRNA levels in the PCa group were generally higher, as compared than those in the BPH group (Fig. 2A). A total of 102 miRNAs were detected in the PCa group, whereas 78 miRNAs were detected in the $\mathrm{BPH}$ group. Of these, 73 miRNAs were detected in both groups (Fig. 2B). This dataset was retained for subsequent processing and differential expression analysis. As there is no standard method for normalization of miRNA expression data in urine samples, various normalization strategies were tested. Rank-invariant set normalization was applied to raw $\mathrm{Ct}$ data, since this method represented the best distribution adjustment to make the two groups of samples comparable, with the exception of quantile normalization. However, the quantile normalization method has been reported to result in a loss of important biological differences (26). Comparison of normalized expression levels of miRNAs revealed a set of 19 miRNAs that showed significant differences between the PCa and BPH groups (Fig. 3). From this set of miRNAs, 11 were elevated, while 8 were reduced in the PCa group. The top ten differentially expressed miRNAs were as follows: miR-100, miR-200b, miR-200c, miR-150, miR-195, let-7b, let-7c, miR-20a, miR-193b and miR-204. This set of miRNAs was selected for further validation in 73 urine samples from patients with $\mathrm{PCa}$ and 70 urine samples from patients with BPH, including the samples used for the initial screening. Significant aberrations were confirmed for miR-100, miR-200b, miR-200c, miR-150, miR-195, let-7b, let-7c and miR-193b (Table II).

Urine levels of miR-100 and miR-200b can be used for distinguishing between PCa and BPH. The urine levels of miR-100 and miR-200b were most significantly increased in patients diagnosed with PCa compared with patients diagnosed with BPH by subsequent biopsy. Since all patients in the PCa group had a Gleason score of $\geq 7$, the difference between indolent and more aggressive PCa could not be assessed. A significant positive correlation was found between the urine levels of miR-100 and miR-200b (Spearman coefficient=0.18; $\mathrm{P}=0.0355$ ) and a unique miR-100/200b score was calculated for further analysis. In the entire cohort, the miR-100/200b score was significantly higher in patients diagnosed with PCa compared with that in patients diagnosed with $\mathrm{BPH}$ by subsequent biopsy (Fig. 4A). The miR-100/200b score was then compared among 
Table I. Clinical features of patients.

\begin{tabular}{|c|c|c|c|}
\hline Variable & Total & $\mathrm{PCa}$ & $\mathrm{BPH}$ \\
\hline Patients, n (\%) & $143(100.0)$ & $73(51.1)$ & $70(48.9)$ \\
\hline Age at diagnosis, mean (range) in years & $66(50-87)$ & $68(51-87)$ & $65(50-82)$ \\
\hline \multicolumn{4}{|l|}{ PSA } \\
\hline Total, mean (range) in $\mathrm{ng} / \mathrm{ml}$ & $12.8(0.9-72.4)$ & $15.7(5-72.4)$ & $9.8(0.9-36)$ \\
\hline Free PSA, mean (range) in $\%$ & $14.4(5-38)$ & $12.5(5-28)$ & $16(5-38)$ \\
\hline$<4 \mathrm{ng} / \mathrm{ml}, \mathrm{n}(\%)$ & $2(1.4)$ & $0(0.0)$ & $2(1.4)$ \\
\hline$>4,<10 \mathrm{ng} / \mathrm{ml}, \mathrm{n}(\%)$ & $77(53.8)$ & $31(21.7)$ & $46(32.2)$ \\
\hline$>10 \mathrm{ng} / \mathrm{ml}, \mathrm{n}(\%)$ & $66(46.2)$ & $42(29.4)$ & $22(15.4)$ \\
\hline \multicolumn{4}{|l|}{ Gleason score, n (\%) } \\
\hline 7 & & $28(19.6)$ & \\
\hline 8 & & $33(23.1)$ & \\
\hline 9 & & $2(1.4)$ & \\
\hline 10 & & $10(7.0)$ & \\
\hline \multicolumn{4}{|l|}{ Suspicious DRE result, n (\%) } \\
\hline Yes & $68(47.6)$ & $47(32.9)$ & $21(14.7)$ \\
\hline No & $75(52.4)$ & $26(18.2)$ & $49(34.3)$ \\
\hline \multicolumn{4}{|l|}{ Lymph node metastasis, n (\%) } \\
\hline No & & $63(44.0)$ & \\
\hline $\mathrm{N} 1$ & & $3(2.1)$ & \\
\hline Unknown & & $7(4.9)$ & \\
\hline
\end{tabular}

PCa, prostate cancer; BPH, benign prostate hyperplasia; PSA, prostate-specific antigen; DRE, digital rectal examination.

patients with suspicion of PCa with PSA levels in the 'gray area' (4-10 $\mathrm{ng} / \mathrm{ml})$, revealing that the miR-100/200b score was significantly higher in patients with PCa-positive biopsy results compared with those in patients with BPH (Fig. 4B).

Inclusion of the urinary $\mathrm{miR}-100 / 200 \mathrm{~b}$ score enhances the predictive value regarding the prostate biopsy outcome. Age, PSA, percentage of free PSA, DRE and miR-100/200b score were evaluated by univariable and multivariable logistic regression analysis to test their predictive value regarding PCa-associated biopsy results (Table III). For the entire cohort, all variables were significant individual predictors of PCa according to the univariable logistic regression model, with the miR-100/200b score DRE having the highest prediction accuracy, followed by the free PSA, PSA, DRE and age. Subsequently a multivariable logistic regression model comprising age, PSA, percentage of free PSA and DRE (base model) was generated to predict the presence of PCa. Addition of the miR-100/200b score to the base model (combined model) yielded a gain of $6.3 \%$ in prediction accuracy. ROC curve analysis demonstrated that the combined model significantly outperformed the capacity of PSA levels (sensitivity, 0.70; specificity, 0.66; $\mathrm{P}<0.001$ ) and the base model (sensitivity, 0.68; specificity, $0.83 ; \mathrm{P}=0.01$ ) for discriminating between $\mathrm{PCa}$ and $\mathrm{BPH}$ patients, with enhanced sensitivity (0.822) and specificity (0.814) at the optimal cutoff value of the estimated coefficients (Table III; Fig. 5). DCA showed that, across a range of threshold probabilities of interest (10-50\%), the highest net benefit was achieved by using the combined model (Fig. 6). For example, at a threshold probability of $26 \%$, the combined model reached a net benefit of 0.40 , which meant that performing a biopsy based on this model was equivalent to a strategy aimed at identifying 40 cancers per 100 patients without performing any unnecessary biopsies, that is a biopsies with a very high probability of being negative for the presence of cancer cells. At this probability threshold, the combined model produced the higher net reduction of 17 unnecessary biopsies per 100 patients, in comparison with the decision based on the PSA model, which had no net reduction, and the base model, which only produced a net reduction of 7 biopsies. These results suggested that adding the miR-100/200b score to the combined model may reduce the number of biopsies with potentially negative results.

Inclusion of the urinary miR-100/200b score enhances the predictive value regarding the prostate biopsy outcome for patients with PSA levels in the gray zone. For the evaluation of the sub-group of patients with PSA levels in the gray zone, age and $\mathrm{miR}-100 / 200 \mathrm{~b}$ score were independent prognostic factors of $\mathrm{PCa}$, as determined by univariable logistic regression analysis. Multivariable logistic regression analysis showed that the combined model significantly improved the prediction of $\mathrm{PCa}$, compared to that using any of the variables alone, and produced a gain in prediction accuracy of $9.1 \%$ compared to that of the base model (Table IV). ROC analysis indicated that specificity of the combined model (0.956) was not superior to PSA levels (0.957). However, the overall performance of the combined model was significantly superior (sensitivity, 0.677 ; 
A

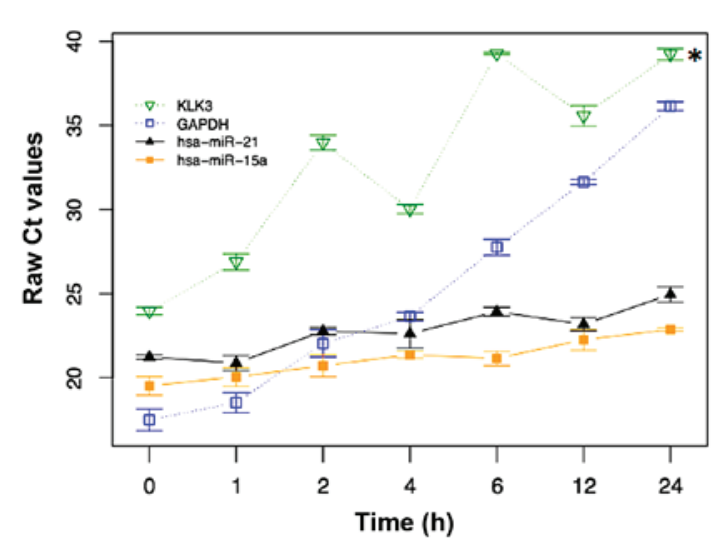

B

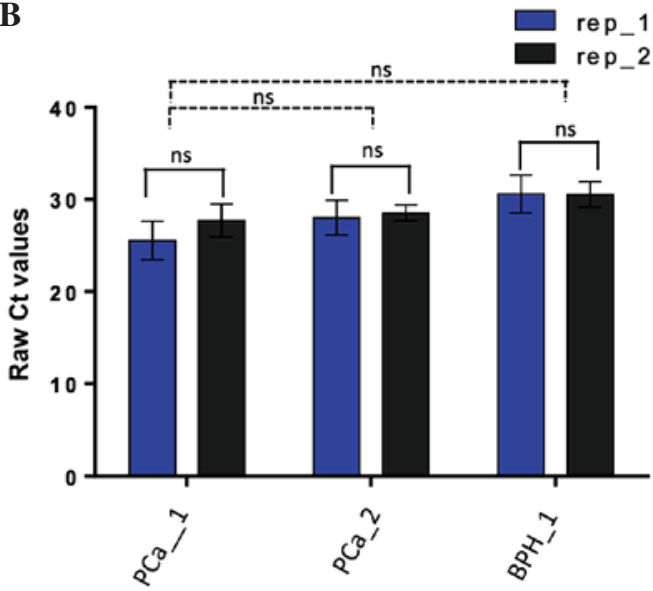

Figure 1. miRNA stability and reproducibility of its quantification in urine samples. (A) Aliquots were removed in triplicate from a pool consisting of 3 PCa and 3 BPH urine samples at 0, 1, 2, 4, 6, 12 and 24 h. miR-15a, miR-21, and KLK3 and GAPDH mRNA, were measured by qPCR and Ct values were obtained. Data are presented as the mean \pm standard deviation of raw $\mathrm{Ct}$ values $(\mathrm{n}=3)$. Comparisons between raw $\mathrm{Ct}$ distributions were conducted using the Friedman test. "P<0.05 vs miR-21. (B) Individual aliquots were removed in duplicate from three urine samples (two PCa patients and one BPH). MiR-15a levels were measured by RT-qPCR performed in triplicate. Data are presented as the mean \pm standard deviation of raw Ct values. miRNA, microRNA; rep, replicate; Ct, cycle threshold. $\mathrm{P}<0.05$ as assessed by the Mann-Whitney U test was considered to indicate a statistically significant difference.

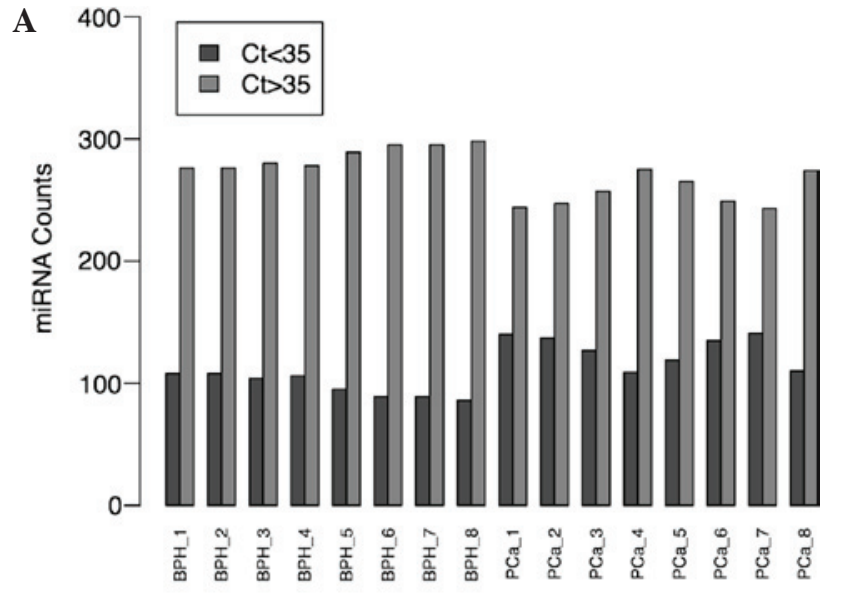

$\mathbf{B}$

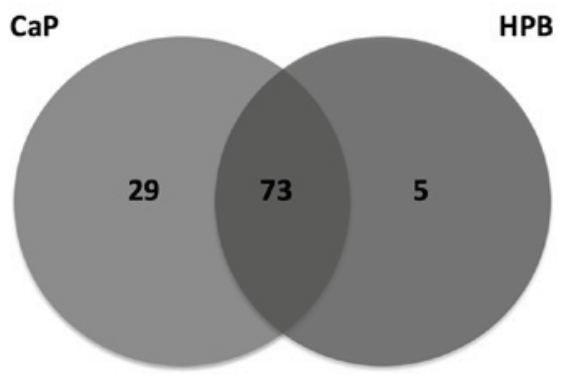

Figure 2. Number of detected and undetected miRNAs in PCa and BPH urine samples. (A) A filtering procedure was applied to classify the evaluated miRNAs according to their level of detectability. A Ct threshold value of 35 was established; a miRNA was considered detected (blue) when $\mathrm{Ct}<35$ and undetected (red) when $\mathrm{Ct}>35$. The number of undetected and detected miRNAs for each patient is plotted on the y-axis. (B) Venn diagram showing the number of miRNAs detected in the PCa group alone, in the BPH group alone and in both groups. miRNA, microRNA; Ct, cycle threshold; PCa, prostate cancer; $\mathrm{BPH}$, benign prostate hyperplasia.

predictive accuracy, 84.4\%), as compared with PSA levels (sensitivity, 0.290; predictive accuracy, 68.8\%; P<0.001) and the base model (sensitivity, 0.548; predictive accuracy, 75.3\%; $\mathrm{P}<0.001$; Fig. 5).

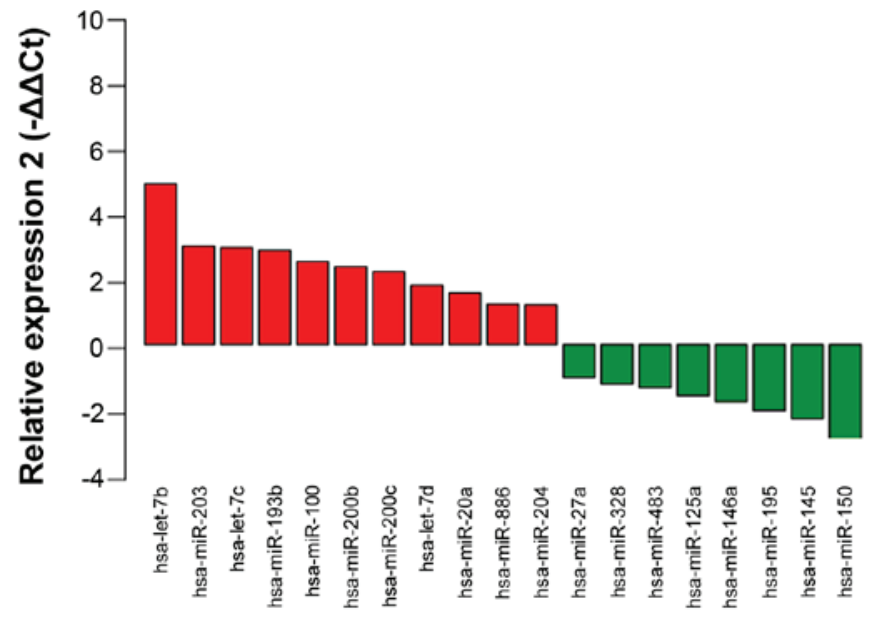

Figure 3. Differentially expressed miRNAs were determined, according to a screening analysis. Fold changes in the PCa group $(n=8)$, as compared with the BPH group $(n=8)$, were computed as $2^{-\Delta \Delta C t}$ and are plotted on the $y$-axis. Significant differences were determined using a Mann Whitney $U$ test with the cutoff value set at $\mathrm{P}<0.05$. miR, microRNA; $\mathrm{Ct}$, cycle threshold.

DCA analysis demonstrated that the combined model produced a greater net benefit for patients with suspicious DRE results who would opt for biopsy at a reasonable threshold probability range of $10-50 \%$, as compared with the use of PSA levels alone or the base model (Fig. 6). At a probability threshold of $26 \%$, the use of the combined model permits the identification 27 cancers per 100 patients, and reduces the number of unnecessary biopsies by 26 per 100 patients. This is in comparison with a decision based on the PSA levels or base model, which produced a net reduction of only 2 and 8 biopsies, respectively. These results indicate that the predictive performance of the miR-100/200b score is similar for the sub-groups of patients within the gray zone and for patients with abnormal DRE results. However, using the miR-100/200b score is advantageous for patients in the gray zone of PSA values. 
Table II. Averaged normalized levels of miRNAs in the urine of patients with PCa and BPH and statistical validation of significant differences.

\begin{tabular}{|c|c|c|c|c|c|}
\hline miRNA & $\Delta \mathrm{Ct}(\mathrm{PCa})$ & $\Delta \mathrm{Ct}(\mathrm{BPH})$ & $\Delta \Delta \mathrm{Ct}$ & Fold change $\left(2^{-\Delta \Delta C t}\right)$ & P-value \\
\hline hsa-miR-100a & 4.2782 & 5.9839 & -1.7058 & 3.2620 & $5.52 \times 10^{-5}$ \\
\hline hsa-miR-200b ${ }^{a}$ & 2.2534 & 4.6239 & -2.3705 & 5.1713 & $2.94 \times 10^{-4}$ \\
\hline hsa-miR-200c a & 2.7771 & 4.1767 & -1.3995 & 2.6382 & $4.60 \times 10^{-4}$ \\
\hline hsa-miR-150 a & 6.6473 & 4.9470 & 1.7003 & 0.3077 & $1.43 \times 10^{-2}$ \\
\hline hsa-miR-195 & 8.9949 & 7.6015 & 1.3934 & 0.3807 & $1.67 \times 10^{-2}$ \\
\hline hsa-let-7ba & 2.6407 & 3.9059 & -1.2652 & 2.4035 & $2.26 \times 10^{-2}$ \\
\hline hsa-let-7ca & 3.2002 & 4.2061 & -1.0058 & 2.0081 & $3.49 \times 10^{-2}$ \\
\hline hsa-miR-20a & 5.5777 & 6.5679 & -0.9901 & 1.9864 & $7.53 \times 10^{-2}$ \\
\hline hsa-miR-193b & 2.8863 & 3.9914 & -1.1051 & 2.1511 & $9.10 \times 10^{-2}$ \\
\hline hsa-miR-204 & 5.6469 & 5.0981 & 0.5487 & 0.6836 & $3.69 \times 10^{-1}$ \\
\hline
\end{tabular}

${ }^{\mathrm{a}} \mathrm{P}<0.05$ according to the two-sample Wilcoxon test indicates that the respective miRNA was successfully validated in a set of independent samples. miRNA, microRNA; Ct, cycle threshold; PCa, prostate cancer; BPH, benign prostate hyperplasia.

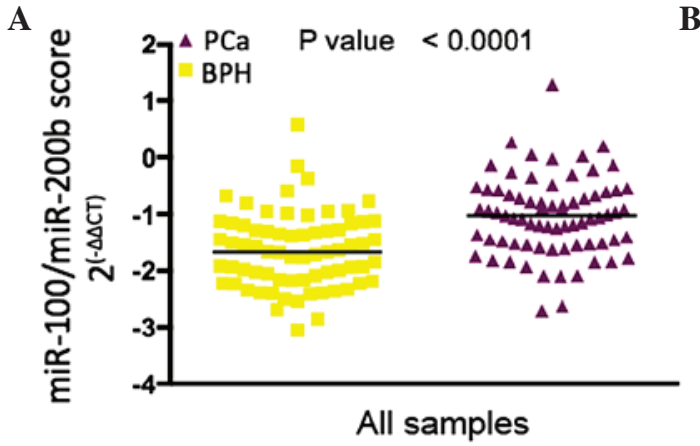

Figure 4. Association of urine levels of miR-100 and miR-200b with the outcome of prostate biopsy. Comparison of the miR-100/miR-200b expression ratio between $\mathrm{PCa}$ and $\mathrm{BPH}$ patients (A) in the entire cohort and (B) in the sub-group with prostate-specific antigen levels in the gray zone. Each data-point shows the value for one patient, and horizontal bars represent the mean value. miR, microRNA; Ct, cycle threshold; PCa, prostate cancer; BPH, benign prostate hyperplasia.

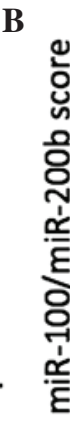

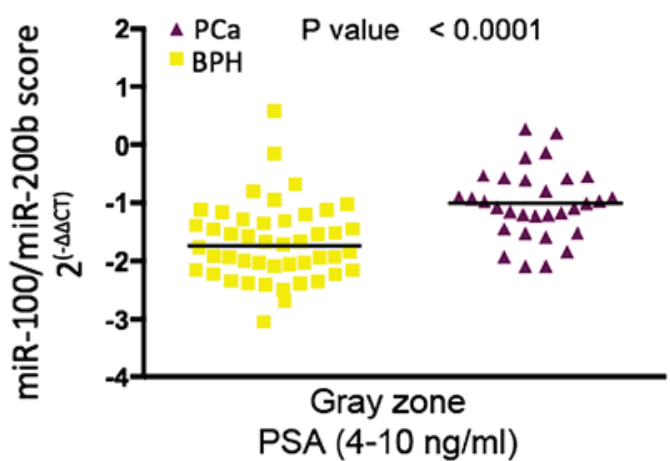

\section{Discussion}

At present, abnormal PSA levels and suspicious DRE results are the main criteria considered for decisions on performing a prostate biopsy (27). In spite of being highly tissue-specific, PSA is not PCa-specific and abnormal serum levels of PSA may also be caused by indolent conditions such as BPH (6). DRE is considered to be a subjective parameter for identifying PCa (28) and almost $50 \%$ of prostate biopsies performed to confirm a suspicion of $\mathrm{PCa}$ are negative and would thus be unnecessary if more precise prediction tools were at hand. Therefore, there is a requirement for novel methods to improve $\mathrm{PCa}$ detection and to therefore minimize the rate of overdiagnosis. Furthermore, it is desired to develop novel diagnostic tools with minimal invasiveness to avoid the comorbidity associated with prostate biopsy. While the risk of unrecognized PCa cannot be completely ruled out (29), a critical aspect of the present study was the identification of patients with $\mathrm{BPH}$ by distinguishing them from patients with $\mathrm{PCa}$ within a cohort with abnormal DRE results. For this set of BPH patients, biopsy specimens were assessed and no evidence of cancer was found. Of note, none of the patients from the BPH group had high-grade prostatic intraepithelial neoplasia or atypical cellular proliferation; therefore, no further biopsy was required. The goal of the present study was to develop a non-invasive method to identify the miRNA signature in the patients' urine to help to distinguish clinically relevant $\mathrm{PCa}$ with a Gleason score of $\geq 7$ from indolent hyperplasia such as $\mathrm{BPH}$.

Urine-based biomarkers are promising indicators of $\mathrm{PCa}$ as they can be obtained by non-invasive methods and allow for monitoring of tumors with heterogeneous foci (30). Recent studies paralleling the present study have demonstrated the feasibility of measuring the levels of miRNAs in urine from PCa patients and comparing them to those of healthy controls or patients with BPH $(19,20,24,31-33)$. The present study confirmed that miRNAs can be readily detected in urine from PCa and BPH patients. In a preliminary attempt, the supernatant fraction remaining after a first centrifugation step was used for miRNA analysis, according to the procedure of a previous study (24). However, in the present study, the RNA yield obtained by this procedure was very low $(<10 \mathrm{ng} / \mu \mathrm{l})$. 


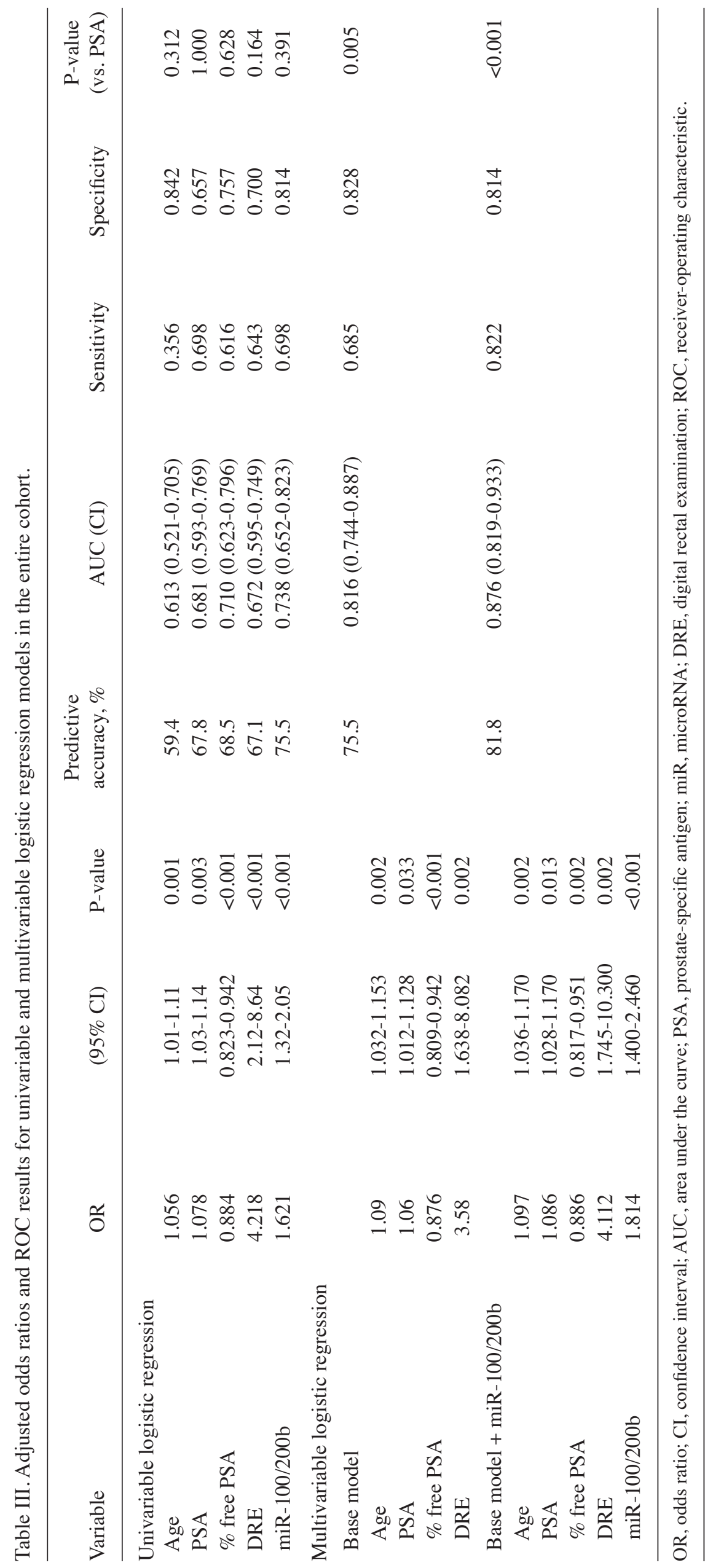




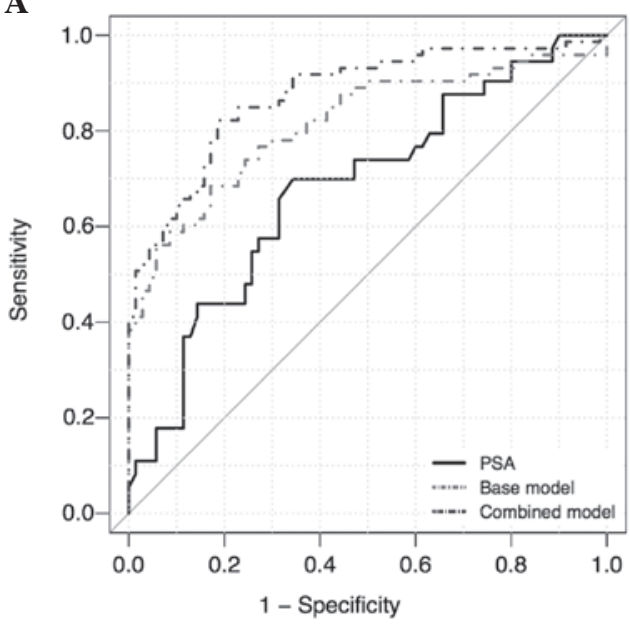

B

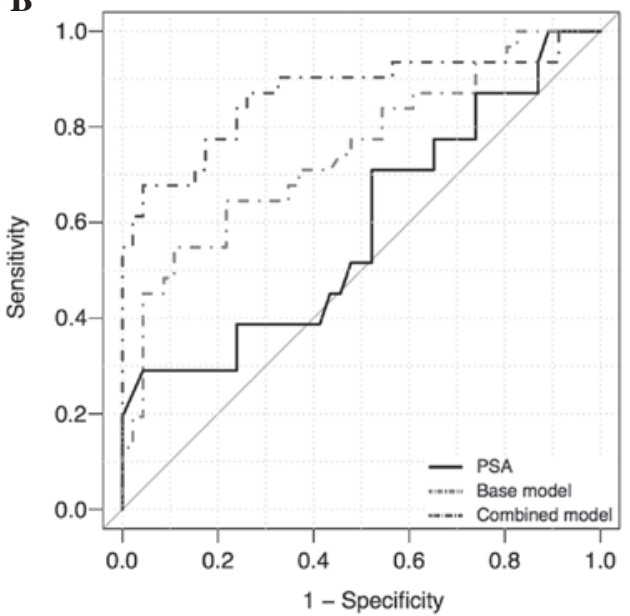

Figure 5. Receiver-operating characteristic analysis for prediction models in (A) the entire cohort and in (B) the subgroup of patients with PSA levels in the gray zone. PSA was used as a reference for comparison. The base model included the following variables: Age, PSA, percentage of free PSA and DRE. The combined model was the base model plus the miR-100/200b score. miR, microRNA; PSA, prostate-specific antigen; DRE, digital rectal examination.
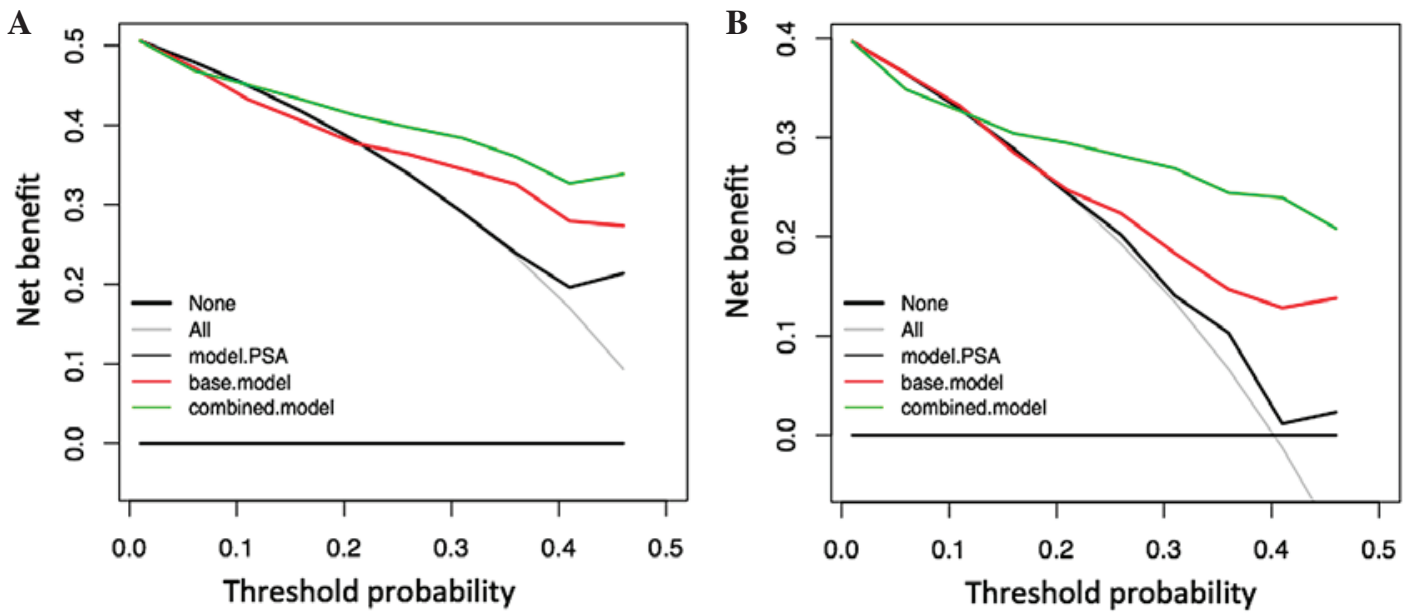

Figure 6. Decision curve analysis of the effects of prediction models on the detection of prostate cancer. (A) Net benefit plotted against various threshold probabilities of undergoing biopsy for patients in the entire cohort. (B) Net benefit plotted against various threshold probabilities of undergoing biopsy for patients in the sub-group of patients with PSA levels in the gray zone. The combined model was the base model plus the miR-100/200b score. PSA, prostate-specific antigen.

Instead, the use of the whole urine sediment yielded higher RNA recovery and improved the detectability of miRNAs by RT-qPCR. To reduce bias, urine specimens were collected and processed as follows: i) Specimens were collected immediately after prostate massage, ii) samples collected were a minimum of $30 \mathrm{ml}$ first-catch urine, iii) specimens with a dark, inhomogeneous appearance and/or with a strong fetor were excluded from the analysis and iv) RNA stabilization agent was added. After establishment of the RT-qPCR detection method and verification of its robustness, comprehensive miRNA profiling was performed with the intention of identifying PCa-specific miRNAs in urine samples. Notably, the overall abundance of miRNAs in urine samples from PCa patients was higher, as compared with urine samples from BPH patients. The reason for this is unclear and, after filtering out miRNAs detected in PCa samples only, the global expression levels of miRNAs in the Pca group were not significantly different, as compared with the BPH group. Brase et al (17) assessed circulating miRNAs and made a similar observation that various miRNAs were highly elevated in the sera of patients with metastatic $\mathrm{PCa}$ in comparison to patients with localized $\mathrm{PCa}$. It is possible that a greater number of miRNA molecules are secreted into the extracellular space with ongoing progression of PCa. In fact, is increasing evidence suggested that PCa cells actively secrete miRNAs and that the profile of the miRNAs secreted dynamically changes as the disease progresses $(33,34)$. These findings further support the use of miRNAs in urine as PCa-specific biomarkers.

The screening analysis of the present study revealed a set of 18 miRNAs whose urine levels were significantly altered in patients with $\mathrm{PCa}(\mathrm{FDR}<0.05 \mathrm{e})$. Among these miRNAs, miR-100 and miR-200b were most significantly upregulated $(\mathrm{P}<0.05)$. Furthermore, a positive correlation between miR-100 and miR-200b was identified; therefore, the miRNA signature was described as the miR-100/200b ratio. In order to measure the degree of correlation between different variables and the outcome of prostate biopsy, an unvariable logistic regression analysis was performed. The results demonstrated that DRE, 


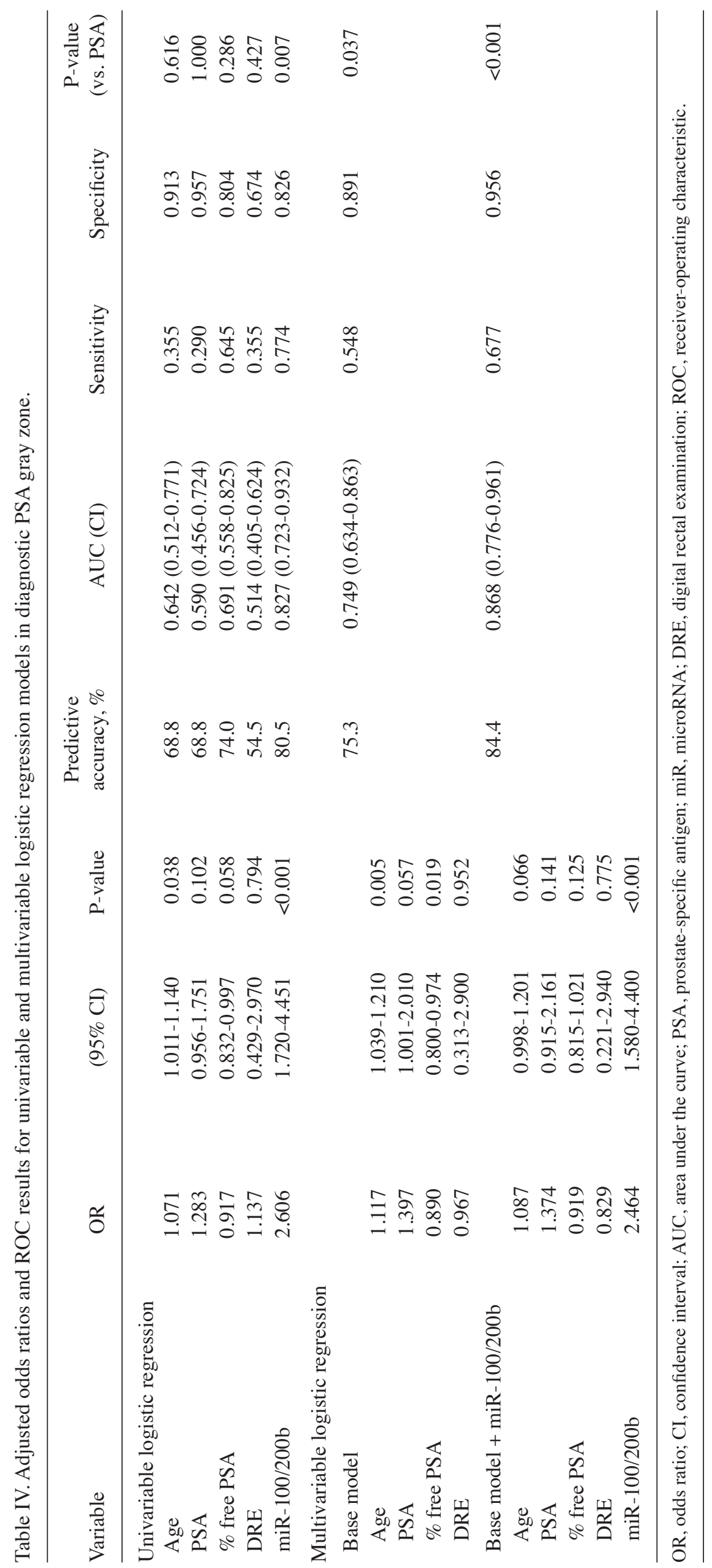


miR-100/200b score, age, PSA and \% free PSA were independent predictors of PCa. Two multivariable models were then constructed: The base model, which comprised DRE, age, PSA and $\%$ free PSA, and the combined model, which comprised all variables in the base model plus the miR-100/200b score. The combined model was a better predictor of the $\mathrm{PCa}$ diagnosis than the miRNA signature alone or any individual clinical parameter. PSA levels have a low predictive value in the diagnostic gray zone $(4-10 \mathrm{ng} / \mathrm{ml})$, and affected patients are currently subjected to prostate biopsy, of which only an estimated $25 \%$ are then diagnosed with PCa (35). Therefore, the use of more specific biomarkers to distinguish patients with $\mathrm{BPH}$ from those with PCa may spare them from unnecessary biopsies. Of note, the results of the present study indicated that, for patients with serum PSA values in the gray zone, the predictive accuracy and performance of the miR-100/200b signature alone was similar to that for the entire cohort. However, the combined model significantly outperformed all other models, indicating that adding the miR-100/200b signature to the base model increased the accuracy of PCa detection within the gray zone, where the clinical decision on whether or not to perform a biopsy remains controversial.

In addition to the assessment of the accuracy in predicting $\mathrm{PCa}$, the clinical benefit of the miRNA signature as part of the combined model was assessed and compared with PSA alone and the base model. For this purpose, DCA was performed, which provided a method allowing for the qualification and identification of the best prediction model to ensure that a biopsy is performed only in patients with a reasonably high probability of positive PCa diagnosis (36). The results showed that the combined model provides a higher net benefit at threshold probabilities of $10-50 \%$, a reasonable range of probabilities to perform a biopsy at the cost of the risk of overtreatment. Of note, the reduction of unnecessary biopsies was maximized within the same threshold probabilities. From a clinical perspective, the performance of the combined model for distinguishing $\mathrm{PCa}$ from $\mathrm{BPH}$ is more crucial in patients with PSA levels in the gray zone. Here, the net benefit was also present within the defined threshold probabilities and a substantial number of potentially negative biopsies may be avoided, providing a greater clinical benefit for this sub-group of patients.

Further molecular markers have been previously evaluated for the diagnosis of PCa. The non-coding RNA PCA3 and the TMPRSS2: ERG gene fusion transcript are the most representative examples of urine biomarkers $(37,38)$. Although PCA3 has been proven to outperform PSA and free \% PSA, the most noteworthy benefit has only been demonstrated in patients who have already undergone biopsies with a negative result (39). However, TMPRSS2: ERG, which can also be detected in urine samples, has a positive predictive value for $\mathrm{PCa}$ of as high as $94 \%$. However, one important drawback in the use of TMPRSS2: ERG is its variable frequency in different populations (6). Despite the efforts to increase the detection rate of $\mathrm{PCa}$, even combining the two transcripts, limitations regarding their sensitivity remain, and their performance is far from being outstanding, particularly at discriminating indolent from significant PCa. One important feature that contributes to the robustness of the use of miRNAs in urine samples as biomarkers is their relatively superior stability for longer time periods in comparison to that of mRNAs (24). The present study was able to detect mRNAs and measure their abundance in the urine samples. However, there are some important limitations of using mRNAs as biomarkers: First, the $\mathrm{Ct}$ values of mRNAs are higher and decay rapidly, as compared with miRNAs; secondly, their $\mathrm{Ct}$ values exhibit a high degree of variation, as was observed for KLK3 in the present study. All of these factors may contribute to the lower reproducibility of mRNAs in urine. Future experiments may be performed to compare the predictive performance of PCA3 and TMPRSS2: ERG with that of the miRNA signature identified in the present study, using the same cohort of patients. In addition, it may be useful to assess the predictive performance of a single model combining all of these biomarkers, with the aim of further enhancing the specificity of the diagnosis.

The present study demonstrated that the elevation of miR-100 and miR-200b levels in urine samples is significantly associated with the presence of advanced PCa. Consistent with this observation, it has been reported that miR-100 is upregulated in high-grade prostate intraepithelial neoplasia, as compared with benign tissue, and remained elevated during the carcinogenic process (40), and that its overexpression was independently correlated with the biochemical recurrence of $\mathrm{PCa}(41)$. In contrast, downregulation of miR-100 has previously been described for hormone-refractory PCa (14). Regarding the presence of miR-100 in biological fluids, a recent study found that miR-100 was upregulated in sera from patients with metastatic castration resistant prostate cancer (mCRPC), as compared with healthy controls. Although miR-100 was not further validated as a predictor marker to mCRPC, the authors showed that miR-100 was among nine miRNAs that were significantly elevated in mCRPC (42). Supporting this notion, Mitchell et al (43) reported an increase in miR-100 expression in the plasma of PCa patients. Furthermore, their increased levels were correlated with poor prognosis. In addition, it has been shown that members of the miR-99 family, including miR-100, have a role in enhancing the sensitivity to radiation in PCa cells (44), reinforcing the value of miR-100 as a marker of PCa.

In conclusion, the present study discovered and validated a urinary miRNA-based signature for enhancing the accuracy of diagnosis of $\mathrm{PCa}$, and to distinguish patients with abnormal DRE results and BPH to spare them from unnecessary biopsy. To the best of our knowledge, the present study was the first describing the use of urinary miR100 and miR-200b levels in combination with clinical parameters to detect the presence of PCa. Similar to the validation process of other biomarkers, this signature requires validation in an independent dataset in order to better evaluate its clinical value.

\section{Acknowledgements}

The authors would like to thank Dr Ignacio Camacho at the Chemistry School of The National Autonomous University of Mexico for reviewing this paper and giving helpful suggestions, and to Dr Sandra Romero-Cordoba at the Oncogenomics Laboratory of The National Institute of Genomic Medicine for technical assistance. The present study was supported by CONACYT (grant nos. 363368 and 177687). Dr Alberto Ivan Salido-Guadarrama wishes to thank the Programa de 
Doctorado en Ciencias Biomédicas-UNAM for providing academic support.

\section{References}

1. Torre LA, Bray F, Siegel RL, Ferlay J, Lortet-Tieulent J and Jemal A: Global cancer statistics, 2012. CA Cancer J Clin 65: 87-108, 2015.

2. De Marzo AM, DeWeese TL, Platz EA, Meeker AK, Nakayama M, Epstein JI, Isaacs WB and Nelson WG: Pathological and molecular mechanisms of prostate carcinogenesis: Implications for diagnosis, detection, prevention, and treatment. J Cell Biochem 91: 459-477, 2004.

3. Schröder FH, Hugosson J, Roobol MJ, Tammela TL, Ciatto S, Nelen V, Kwiatkowski M, Lujan M, Lilja H, Zappa M, et al: Screening and prostate-cancer mortality in a randomized European study. N Engl J Med 360: 1320-1328, 2009.

4. Shappell SB: Clinical utility of prostate carcinoma molecular diagnostic tests. Rev Urol 10: 44-69, 2008.

5. Dall'Era MA, Albertsen PC, Bangma C, Carroll PR, Carter HB Cooperberg MR, Freedland SJ, Klotz LH, Parker C and Soloway MS: Active surveillance for prostate cancer: A systematic review of the literature. Eur Urol 62: 976-983, 2012

6. Romero Otero J, Garcia Gomez B, Campos Juanatey F and Touijer KA: Prostate cancer biomarkers: An update. Urol Oncol 32: 252-260, 2014

7. Mao G, Liu Y, Fang X, Liu Y, Fang L, Lin L, Liu X and Wang N: Tumor-derived microRNA-494 promotes angiogenesis in non-small cell lung cancer. Angiogenesis 18: 373-382, 2015.

8. Kulshreshtha R, Ferracin M, Wojcik SE, Garzon R, Alder H, Agosto-Perez FJ, Davuluri R, Liu CG, Croce CM, Negrini M, et al: A microRNA signature of hypoxia. Mol Cell Biol 27: 1859-1867, 2007.

9. Katz B, Reis ST, Viana NI, Morais DR, Moura CM, Dip N, Silva IA, Iscaife A, Srougi M and Leite KR: Comprehensive study of gene and microRNA expression related to epithelial-mesenchymal transition in prostate cancer. PLoS One 9: e113700, 2014

10. Wang Y, Kim S and Kim IM: Regulation of Metastasis by microRNAs in Ovarian Cancer. Front Oncol 4: 143, 2014.

11. Bouyssou JM, Manier S, Huynh D, Issa S, Roccaro AM and Ghobrial IM: Regulation of microRNAs in cancer metastasis. Biochim Biophys Acta 1845: 255-265, 2014.

12. Fang YX and Gao WQ: Roles of microRNAs during prostatic tumorigenesis and tumor progression. Oncogene 33: 135-147, 2014.

13. Ambs S, Prueitt RL, Yi M, Hudson RS, Howe TM, Petrocca F, Wallace TA, Liu CG, Volinia S, Calin GA, et al: Genomic profiling of microRNA and messenger RNA reveals deregulated microRNA expression in prostate cancer. Cancer Res 68: 6162-6170, 2008.

14. Porkka KP, Pfeiffer MJ, Waltering KK, Vessella RL, Tammela TL and Visakorpi T: MicroRNA expression profiling in prostate cancer. Cancer Res 67: 6130-6135, 2007.

15. Salido-Guadarrama I, Romero-Cordoba S, Peralta-Zaragoza O, Hidalgo-Miranda A and Rodriguez-Dorantes M: MicroRNAs transported by exosomes in body fluids as mediators of intercellular communication in cancer. Onco Targets Ther 7: 1327-1338, 2014.

16. Moltzahn F, Olshen AB, Baehner L, Peek A, Fong L, Stöppler H, Simko J, Hilton JF, Carroll P and Blelloch R: Microfluidic-based multiplex qRT-PCR identifies diagnostic and prognostic microRNA signatures in the sera of prostate cancer patients. Cancer Res 71: 550-560, 2011.

17. Brase JC, Johannes M, Schlomm T, Fälth M, Haese A, Steuber T, Beissbarth T, Kuner R and Sültmann H: Circulating miRNAs are correlated with tumor progression in prostate cancer. Int $\mathrm{J}$ Cancer 128: 608-616, 2011.

18. Park NJ, Zhou H, Elashoff D, Henson BS, Kastratovic DA, Abemayor E and Wong DT: Salivary microRNA: Discovery, characterization, and clinical utility for oral cancer detection. Clin Cancer Res 15: 5473-5477, 2009.

19. Srivastava A, Goldberger H, Dimtchev A, Ramalinga M, Chijioke J, Marian C, Oermann EK, Uhm S, Kim JS, Chen LN, et al: MicroRNA profiling in prostate cancer-the diagnostic potential of urinary miR-205 and miR-214. PLoS One 8: e76994, 2013.

20. Sapre N, Hong MK, Macintyre G, Lewis H, Kowalczyk A, Costello AJ, Corcoran NM and Hovens CM: Curated microRNAs in urine and blood fail to validate as predictive biomarkers for high-risk prostate cancer. PLoS One 9: e91729, 2014.
21. Eissa S, Habib H, Ali E and Kotb Y: Evaluation of urinary miRNA-96 as a potential biomarker for bladder cancer diagnosis. Med Oncol 32: 413, 2015.

22. Haj-Ahmad TA, Abdalla MA and Haj-Ahmad Y: Potential urinary miRNA biomarker candidates for the accurate detection of prostate cancer among benign prostatic hyperplasia patients. J Cancer 5: 182-191, 2014.

23. Groskopf J, Aubin SM, Deras IL, Blase A, Bodrug S, Clark C, Brentano S, Mathis J, Pham J, Meyer T, et al: APTIMA PCA3 molecular urine test: Development of a method to aid in the diagnosis of prostate cancer. Clin Chem 52: 1089-1095, 2006.

24. Lewis H, Lance R, Troyer D, Beydoun H, Hadley M, Orians J, Benzine T, Madric K, Semmes OJ, Drake R and Esquela-Kerscher A: miR-888 is an expressed prostatic secretions-derived microRNA that promotes prostate cell growth and migration. Cell Cycle 13: 227-239, 2014.

25. Livak KJ and Schmittgen TD: Analysis of relative gene expression data using real-time quantitative PCR and the 2(-Delta Delta C(T)) Method. Methods 25: 402-408, 2001

26. Suyundikov A Stevens JR, Corcoran C, Herrick J, Wolff RK and Slattery ML: Incorporation of subject-level covariates in quantile normalization of miRNA data. BMC Genomics 16: 1045,2015

27. Ukimura O, Coleman JA, de la Taille A, Emberton M, Epstein JI, Freedland SJ, Giannarini G, Kibel AS, Montironi R, Ploussard G, et al: Contemporary role of systematic prostate biopsies: Indications, techniques, and implications for patient care. Eur Urol 63: 214-230, 2013.

28. Loeb S, Han M, Roehl KA, Antenor JA and Catalona WJ: Accuracy of prostate weight estimation by digital rectal examination versus transrectal ultrasonography. J Urol 173: 63-65, 2005.

29. Levy DA and Jones JS: Management of rising prostate-specific antigen after a negative biopsy. Curr Urol Rep 12: 197-202, 2011.

30. Jamaspishvili T, Kral M, Khomeriki I, Student V, Kolar Z and Bouchal J: Urine markers in monitoring for prostate cancer. Prostate Cancer Prostatic Dis 13: 12-19, 2010.

31. Korzeniewski N, Tosev G, Pahernik S, Hadaschik B, Hohenfellner $M$ and Duensing S: Identification of cell-free microRNAs in the urine of patients with prostate cancer. Urol Oncol 33: 16.e17-e22, 2015.

32. Stephan C, Jung M, Rabenhorst S, Kilic E and Jung K: Urinary miR-183 and miR-205 do not surpass PCA3 in urine as predictive markers for prostate biopsy outcome despite their highly dysregulated expression in prostate cancer tissue. Clin Chem Lab Med 53: 1109-1118, 2015.

33. Kim J, Morley S, Le M, Bedoret D, Umetsu DT, Di Vizio D and Freeman MR: Enhanced shedding of extracellular vesicles from amoeboid prostate cancer cells: Potential effects on the tumor microenvironment. Cancer Biol Ther 15: 409-418, 2014.

34. Hessvik NP, Phuyal S, Brech A, Sandvig K and Llorente A: Profiling of microRNAs in exosomes released from PC-3 prostate cancer cells. Biochim Biophys Acta 1819: 1154-1163, 2012.

35. Schröder FH, Raaijmakers R, Postma R, van der Kwast TH and Roobol MJ: 4-year prostate specific antigen progression and diagnosis of prostate cancer in the European randomized study of screening for prostate cancer, section Rotterdam. J Urol 174: 489-494; discussion 493-494, 2005

36. Fitzgerald M, Saville BR and Lewis RJ: Decision curve analysis. JAMA 313: 409-410, 2015.

37. Tomlins SA: Urine PCA3 and TMPRSS2: ERG using cancer-specific markers to detect cancer. Eur Urol 65: 543-545, 2014.

38. Cornu JN, Cancel-Tassin G, Egrot C, Gaffory C, Haab F and Cussenot O: Urine TMPRSS2: ERG fusion transcript integrated with PCA3 score, genotyping, and biological features are correlated to the results of prostatic biopsies in men at risk of prostate cancer. Prostate 73: 242-249, 2013

39. Roobol MJ, Schröder FH, van Leeuwen P, Wolters T, van den Bergh RC, van Leenders GJ and Hessels D: Performance of the prostate cancer antigen 3 (PCA3) gene and prostate-specific antigen in prescreened men: Exploring the value of PCA3 for a first-line diagnostic test. Eur Urol 58: 475-481, 2010.

40. Leite KR, Tomiyama A, Reis ST, Sousa-Canavez JM, Sañudo A, Camara-Lopes LH and Srougi M: MicroRNA expression profiles in the progression of prostate cancer - from high-grade prostate intraepithelial neoplasia to metastasis. Urol Oncol 31: 796-801, 2013 
41. Leite KR, Tomiyama A, Reis ST, Sousa-Canavez JM, Sañudo A, Dall'Oglio MF, Camara-Lopes LH and Srougi M: MicroRNA-100 expression is independently related to biochemical recurrence of prostate cancer. J Urol 185: 1118-1122, 2011.

42. Cheng HH, Mitchell PS, Kroh EM, Dowell AE, Chéry L, Siddiqui J, Nelson PS, Vessella RL, Knudsen BS, Chinnaiyan AM, et al: Circulating microRNA profiling identifies a subset of metastatic prostate cancer patients with evidence of cancer-associated hypoxia. PLoS One 8: e69239, 2013.
43. Mitchell PS, Parkin RK, Kroh EM, Fritz BR, Wyman SK, Pogosova-Agadjanyan EL, Peterson A, Noteboom J, O'Briant KC, Allen A, et al: Circulating microRNAs as stable blood-based markers for cancer detection. Proc Natl Acad Sci USA 105: 10513-10518, 2008.

44. Mueller AC, Sun D and Dutta A: The miR-99 family regulates the DNA damage response through its target $\mathrm{SNF} 2 \mathrm{H}$. Oncogene 32: 1164-1172, 2013. 